\title{
Engineering Green Lubricants II: Thermal \\ Transition and Flow Properties of Vegetable Oil- Derived Diesters
}

\author{
Latchmi Raghunanan and Suresh S. Narine." \\ Trent Centre for Biomaterials Research, Departments of Physics \& Astronomy and Chemistry, Trent University, \\ 1600 West Bank Drive, Peterborough, ON, Canada K9L 0G2 \\ *Corresponding Author: Fax: 705750 2786; Tel: 705748 1011; E-mail: sureshnarine@trentu.ca
}

Total number of pages: 11

Table S.1: Nomenclature and structural characterization of the linear diesters synthesized in this work. .............................................. S2

Scheme S.1: Synthesis of symmetric diesters from fatty acids and diols.................. S7

Scheme S.2: Showing the two-stage syntheses of asymmetric diesters. ...................... S7

Figure S.1: DSC cooling $\left(3{ }^{\circ} \mathrm{C} / \mathrm{min}\right)$ thermograms of the linear aliphatic diesters. ........ S8

Figure S.2: DSC heating $\left(3{ }^{\circ} \mathrm{C} / \mathrm{min}\right)$ thermograms of linear aliphatic diesters. ............. S8

Figure S.3: (a) WAXD plots for 22-4-22 and 22-9-22 diesters obtained at $0{ }^{\circ} \mathrm{C}$ and -25 ${ }^{\circ} \mathrm{C}$, respectively using a cooling rate of $1{ }^{\circ} \mathrm{C} / \mathrm{min}$. (b) DSC cooling profiles of 22-4-22 and 22-9-22 obtained using the same thermal protocol as was used for determination of the WAXD profiles.

Figure S.4: Shear rate versus shear stress plots of representative diester series measured at $40{ }^{\circ} \mathrm{C}$ (a) 10 -n-10 and (b) 22 -n-22 diesters.

Figure S.5: Temperature-Viscosity profiles of representative diester series (a) 10-n-10; and (b) 22-n-22. 
Table S.1: Nomenclature and structural characterization of the linear diesters synthesized in this work.

\begin{tabular}{|c|c|c|}
\hline Compound & IUPAC Name & Structure Characterization \\
\hline \multicolumn{3}{|c|}{ Symmetric unsaturated diesters: } \\
\hline $10-2-10$ & $\begin{array}{l}\text { ethane-1,2-diyl bis(dec-9- } \\
\text { enoate) }\end{array}$ & $\begin{array}{l}\mathrm{IR}(\mathrm{ATR}): 1735(\mathrm{C}=\mathrm{O}) \mathrm{cm}^{-1} ;{ }^{1} \mathrm{H}-\mathrm{NMR} \text { in } \mathrm{CDCl}_{3} \delta(\mathrm{ppm}): 1.27-1.32 \\
\left(12, \mathrm{~m}, \mathrm{C}-\mathrm{CH}_{2}-\right), 1.34-1.39\left(4, \mathrm{~m}, \mathrm{H}_{2} \mathrm{C}=\mathrm{CCCH}_{2}-\right), 1.58-1.63(4, \mathrm{~m}, \\
\left.\mathrm{O}=\mathrm{CCCH}_{2}-\right), 2.01-2.05\left(4, \mathrm{q}, \mathrm{H}_{2} \mathrm{C}=\mathrm{CCCH}_{2}-\right), 2.30-2.33(4, \mathrm{t}, \\
\left.\mathrm{O}=\mathrm{CC} \underline{\mathrm{H}}_{2}-\right), 4.26\left(4, \mathrm{~s}, \mathrm{O}-\mathrm{CH}_{2}-\right), 4.91-5.00\left(4, \mathrm{qd},-\mathrm{HC}=\mathrm{C}_{2}\right), 5.75- \\
5.83\left(2, \mathrm{~m}, \mathrm{H}_{2} \mathrm{C}=\mathrm{C} \underline{H}_{-}-\right) . \mathrm{MS}(\mathrm{ESI}): \text { calculated for } \mathrm{C}_{22} \mathrm{H}_{38} \mathrm{O}_{4} 367, \text { found } \\
m / z 390\left([\mathrm{M}+\mathrm{Na}]^{+}\right) .\end{array}$ \\
\hline $10-3-10$ & $\begin{array}{l}\text { propane-1,3-diyl bis(dec- } \\
\text { 9-enoate) }\end{array}$ & 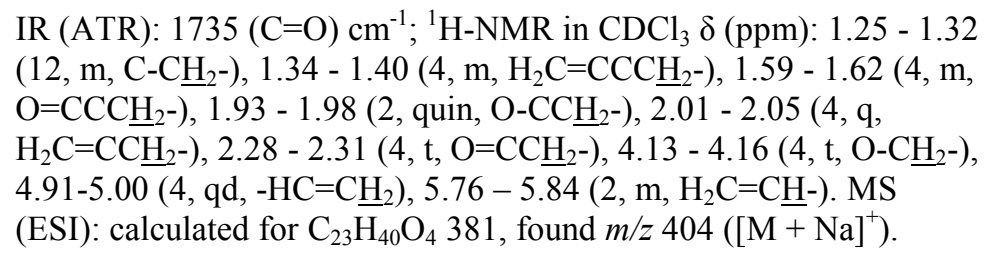 \\
\hline $10-4-10$ & $\begin{array}{l}\text { butane-1,4-diyl bis(dec-9- } \\
\text { enoate) }\end{array}$ & 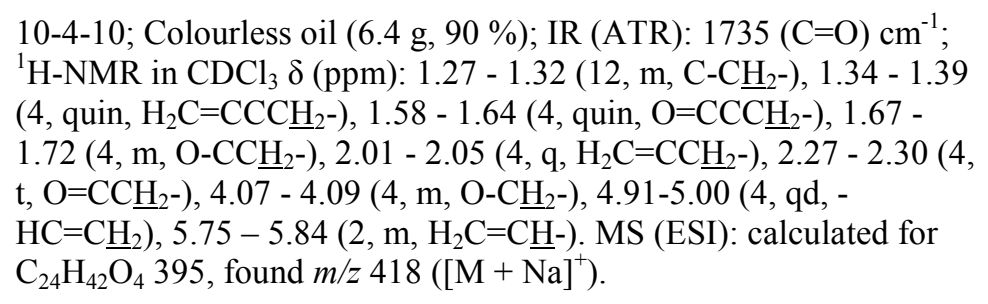 \\
\hline $10-6-10$ & $\begin{array}{l}\text { hexane-1,6-diyl bis(dec- } \\
\text { 9-enoate) }\end{array}$ & 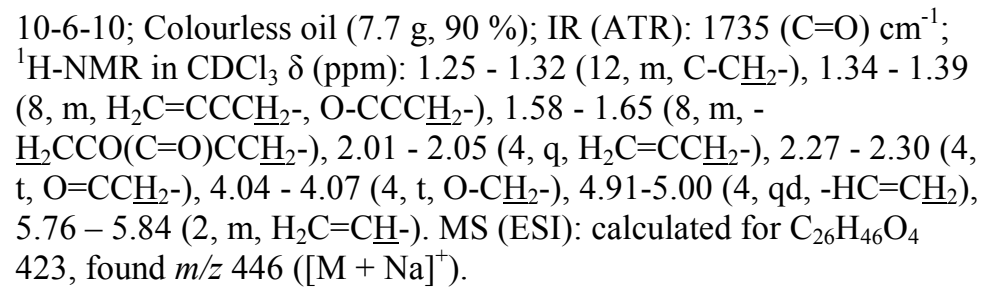 \\
\hline $18-2-18$ & ethane-1,2-diyl dioleate & $\begin{array}{l}\text { IR (ATR): } 1742(\mathrm{C}=\mathrm{O}) \mathrm{cm}^{-1} ;{ }^{1} \mathrm{H}-\mathrm{NMR} \text { in } \mathrm{CDCl}_{3} \delta(\mathrm{ppm}): 5.27-5.36 \\
(4, \mathrm{~m},-\mathrm{CH}=\mathrm{C} \underline{\mathrm{H}}-), 4.24\left(4, \mathrm{~s}, \mathrm{O}-\mathrm{CH}_{2}-\right), 2.27-2.31\left(4, \mathrm{t}, \mathrm{O}=\mathrm{CC} \underline{\mathrm{H}}_{2}-\right) \\
1.96-2.00\left(8, \mathrm{~m}, \mathrm{C}=\mathrm{CC} \underline{\mathrm{H}}_{2}-\right), 1.58-1.61\left(4, \mathrm{~m}, \mathrm{O}=\mathrm{CCC} \underline{\mathrm{H}}_{2}-\right), 1.24-1.28 \\
\left(40, \mathrm{~m}, \mathrm{C}-\mathrm{C}_{2}-\right), 0.84-0.87\left(6, \mathrm{t}, \mathrm{C}-\mathrm{C}_{3}\right) . \mathrm{MS}(\mathrm{ESI}): \text { calculated for } \\
\mathrm{C}_{38} \mathrm{H}_{70} \mathrm{O}_{4} 590, \text { found } \mathrm{m} / \mathrm{z} 608\left(\left[\mathrm{M}+\mathrm{NH}_{4}\right]^{+}\right) .\end{array}$ \\
\hline $18-3-18$ & propane-1,3-diyl dioleate & $\begin{array}{l}\text { IR (ATR): } 1739(\mathrm{C}=\mathrm{O}) \mathrm{cm}^{-1} ;{ }^{1} \mathrm{H}-\mathrm{NMR} \text { in } \mathrm{CDCl}_{3} \delta(\mathrm{ppm}): 5.28-5.36 \\
(4, \mathrm{~m},-\mathrm{CH}=\mathrm{CH}-), 4.11-4.14\left(4, \mathrm{t}, \mathrm{O}-\mathrm{C}_{\mathrm{H}_{2}}\right), 2.25-2.29(4, \mathrm{~m}, \\
\left.\mathrm{O}=\mathrm{CC} \underline{\mathrm{H}}_{2}-\right), 1.94-2.03\left(8 \mathrm{H}, \mathrm{m},-\mathrm{CH}=\mathrm{CC}_{\mathrm{H}_{2}}\right), 1.91-1.92(2 \mathrm{H}, \mathrm{m}, \mathrm{O}- \\
\left.\mathrm{CC} \underline{\mathrm{H}}_{2}-\right), 1.55-1.63\left(4 \mathrm{H}, \mathrm{m}, \mathrm{O}=\mathrm{CCC} \underline{\mathrm{H}}_{2}-\right), 1.20-1.35\left(40, \mathrm{~m}, \mathrm{C}-\mathrm{C} \underline{\mathrm{H}}_{2}-\right), \\
0.83-0.88\left(6, \mathrm{t}, \mathrm{C}-\mathrm{CH} \underline{\mathrm{H}}_{3}\right) . \mathrm{MS}(\mathrm{ESI}): \text { calculated for } \mathrm{C}_{39} \mathrm{H}_{72} \mathrm{O}_{4} 604, \\
\text { found } m / z 622\left(\left[\mathrm{M}+\mathrm{NH}_{4}\right]^{+}\right) \text {. }\end{array}$ \\
\hline $18-4-18$ & butane-1,4-diyl dioleate & 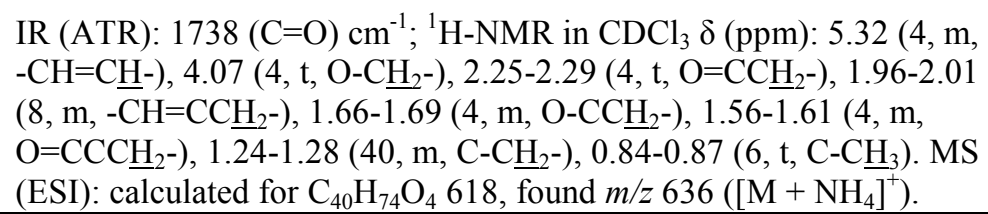 \\
\hline
\end{tabular}


18-6-18 hexane-1,6-diyl dioleate

18-9-18 nonane-1,9-diyl dioleate

18-10-18 decane-1,10-diyl dioleate
IR (ATR): $1737(\mathrm{C}=\mathrm{O}) \mathrm{cm}^{-1} ;{ }^{1} \mathrm{H}-\mathrm{NMR}$ in $\mathrm{CDCl}_{3} \delta$ (ppm): 5.25-5.33 $\left(4, \mathrm{~m},-\mathrm{CH}=\mathrm{CH}-\right.$ ), 3.99-4.03 (4, t, O- $\left.\underline{\mathrm{H}}_{2}-\right), 2.22-2.26\left(4, \mathrm{t}, \mathrm{O}=\mathrm{CCH}_{2}-\right.$ ), 1.95-1.97 (8, m, $\left.-\mathrm{CH}=\mathrm{CCH}_{2}-\right), 1.55-1.60$ (8, m, O- $\mathrm{CCH}_{2}-$, $\left.\mathrm{O}=\mathrm{CCC} \underline{\mathrm{H}}_{2}-\right), 1.21-1.35$ (44, m, C-C $\left.\underline{\mathrm{H}}_{2}-\right), 0.82-0.85$ (6, t, C-C $\left.\underline{\mathrm{H}}_{3}\right) . \mathrm{MS}$ (ESI): calculated for $\mathrm{C}_{42} \mathrm{H}_{78} \mathrm{O}_{4} 646$, found $m / z 664\left(\left[\mathrm{M}+\mathrm{NH}_{4}\right]^{+}\right)$.

IR (ATR): $1737(\mathrm{C}=\mathrm{O}) \mathrm{cm}^{-1} ;{ }^{1} \mathrm{H}-\mathrm{NMR}$ in $\mathrm{CDCl}_{3} \delta(\mathrm{ppm}):$ 5.31-5.33 $\left(4, \mathrm{~m},-\mathrm{CH}=\mathrm{CH}-\underline{-}^{-}, 4.01-4.05\left(4, \mathrm{t}, \mathrm{O}-\mathrm{CH}_{2}-\right), 2.24-2.26\left(4, \mathrm{t}, \mathrm{O}=\mathrm{CCH}_{2}-\right.\right.$ ), 1.96-2.01 (8, m, $\left.-\mathrm{CH}=\mathrm{CCH}_{2}-\right), 1.56-1.61\left(8, \mathrm{~m}, \mathrm{O}-\mathrm{CC}_{2}{ }_{2}-\right.$, $\left.\mathrm{O}=\mathrm{CCCH}_{2}-\right), 1.24-1.29\left(50, \mathrm{~m}, \mathrm{C}-\mathrm{CH}_{2}-\right), 0.84-0.87\left(6, \mathrm{t}, \mathrm{C}-\mathrm{CH}_{3}\right) . \mathrm{MS}$ (ES): calculated for $\mathrm{C}_{45} \mathrm{H}_{84} \mathrm{O}_{4} 688$, found $m / z 706\left(\left[\mathrm{M}+\mathrm{NH}_{4}\right]^{+}\right)$.

IR (ATR): $1737(\mathrm{C}=\mathrm{O}) \mathrm{cm}^{-1} ;{ }^{1} \mathrm{H}-\mathrm{NMR}$ in $\mathrm{CDCl}_{3} \delta(\mathrm{ppm}): 5.27-5.35$ $(4, \mathrm{~m},-\mathrm{CH}=\mathrm{CH}-), 4.00-4.04\left(4, \mathrm{t}, \mathrm{O}-\mathrm{CH}_{2}-\right), 2.23-2.27\left(4, \mathrm{t}, \mathrm{O}=\mathrm{CCH}_{2}-\right.$ ), 1.95-2.00 (8, m, $\left.-\mathrm{CH}=\mathrm{CCH}_{2}-\right), 1.55-1.60\left(8, \mathrm{~m}, \mathrm{O}-\mathrm{CCH}_{2-}\right.$, $\left.\mathrm{O}=\mathrm{CCC}_{\mathrm{H}_{2}}-\right), 1.24-1.27\left(52, \mathrm{~m}, \mathrm{C}-\mathrm{C}_{2}-\right), 0.83-0.87\left(6, \mathrm{t}, \mathrm{C}-\mathrm{CH}_{3}\right) . \mathrm{MS}$ (ESI): calculated for $\mathrm{C}_{46} \mathrm{H}_{86} \mathrm{O}_{4} 702$, found $m / z 720\left(\left[\mathrm{M}+\mathrm{NH}_{4}\right]^{+}\right)$.

IR (ATR): $1721(\mathrm{C}=\mathrm{O}) \mathrm{cm}^{-1} ;{ }^{1} \mathrm{H}-\mathrm{NMR}$ in $\mathrm{CDCl}_{3} \delta(\mathrm{ppm}): 0.78-0.93$ (6, m, C-C $\left.\underline{H}_{3}\right), 1.16$ - 1.40 (56, m, C-C $\left.\underline{\mathrm{H}}_{2}-\right), 1.51$ - 1.68 (4, m, $\left.\mathrm{O}=\mathrm{CCC}^{-} \underline{H}_{2}\right), 1.92$ - $1.97\left(2, \mathrm{~m}, \mathrm{O}-\mathrm{CC}_{\mathrm{H}_{2}}-\right), 1.97$ - 2.05 (8, m, $\left.\mathrm{CH}=\mathrm{CCH}_{2}-\right), 2.29\left(4, \mathrm{t}, \mathrm{O}=\mathrm{CCH}_{2}-\right), 4.14\left(4, \mathrm{t}, \mathrm{O}-\mathrm{CH}_{2}-\right), 5.34$ (4, t, $\mathrm{CH}=\mathrm{CH}-$ ). MS (ESI): calculated for $\mathrm{C}_{47} \mathrm{H}_{88} \mathrm{O}_{4} 717$, found $m / z 740$ $\left([\mathrm{M}+\mathrm{Na}]^{+}\right)$.

IR (ATR): $1723(\mathrm{C}=\mathrm{O}) \mathrm{cm}^{-1} ;{ }^{1} \mathrm{H}-\mathrm{NMR}$ in $\mathrm{CDCl}_{3} \delta(\mathrm{ppm}): 0.78-1.01$ (6, m, C- $\left.\underline{\mathrm{CH}}_{3}\right), 1.17$ - 1.39 (56, m, C-C $\left.\underline{\mathrm{H}}_{2}-\right), 1.51$ - 1.65 (4, m, $\left.\mathrm{O}=\mathrm{CCC}_{\mathrm{H}_{2}}{ }^{-}\right), 1.65-1.77\left(4, \mathrm{~m}, \mathrm{O}-\mathrm{CC}_{\mathrm{H}_{2}}-\right), 1.91-2.11(8, \mathrm{~m},-$

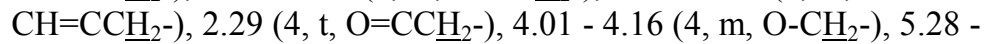
$5.52(4, \mathrm{~m},-\mathrm{CH}=\mathrm{CH}-)$. MS (ESI): calculated for $\mathrm{C}_{48} \mathrm{H}_{90} \mathrm{O}_{4} 731$, found $m / z 754\left([\mathrm{M}+\mathrm{Na}]^{+}\right)$.

IR (ATR): $1725(\mathrm{C}=\mathrm{O}) \mathrm{cm}^{-1} ;{ }^{1} \mathrm{H}-\mathrm{NMR}$ in $\mathrm{CDCl}_{3} \delta$ (ppm): $0.72-0.96$ (6, m, C- $\left.\underline{\mathrm{H}}_{3}\right), 1.20$ - 1.35 (56, m, C- $\left.\underline{\mathrm{H}}_{2}-\right), 1.38$ (4, dt, O-CCC $\underline{\mathrm{H}}_{2}-$ ), 1.53 - $1.71\left(8, \mathrm{~m}, \mathrm{O}=\mathrm{CCCH}_{2}-, \mathrm{O}-\mathrm{CCH}_{2}-\right), 1.92-2.09$ (8, m, $\left.\mathrm{CH}=\mathrm{CCH}_{2}-\right), 2.28\left(4, \mathrm{t}, \mathrm{O}=\mathrm{CCH}_{2}-\right), 4.05$ (4, t, O- $\left.\underline{C H}_{2}-\right), 5.34$ (4, t, $\mathrm{CH}=\mathrm{CH}-$ ). MS (ESI): calculated for $\mathrm{C}_{50} \mathrm{H}_{94} \mathrm{O}_{4} 759$, found $\mathrm{m} / z 782$ $\left([\mathrm{M}+\mathrm{Na}]^{+}\right)$.

IR (ATR): $1725(\mathrm{C}=\mathrm{O}) \mathrm{cm}^{-1} ;{ }^{1} \mathrm{H}-\mathrm{NMR}$ in $\mathrm{CDCl}_{3} \delta$ (ppm): $0.80-0.96$ (6, m, C-C $\left.\underline{H}_{3}\right), 1.17$ - $1.41\left(66, \mathrm{~m}, \mathrm{C}-\mathrm{C}_{2}-\right), 1.61$ (8, quin, $\left.\mathrm{O}=\mathrm{CCCH}_{2}-, \mathrm{O}-\mathrm{CC}_{2_{2}}-\right), 1.90-2.09\left(8, \mathrm{~m},-\mathrm{CH}=\mathrm{CC}_{\mathrm{H}_{2}}-\right), 2.28$ (4, t, $\left.\mathrm{O}=\mathrm{CC}_{2_{2}}-\right), 4.05$ (4, t, O- $\left.\underline{\mathrm{H}}_{2}-\right), 5.29$ - 5.42 (4, m, - $\left.\mathrm{CH}=\mathrm{C} \underline{\mathrm{H}}-\right)$. MS (ESI): calculated for $\mathrm{C}_{53} \mathrm{H}_{100} \mathrm{O}_{4} 801$, found $m / z 802\left([\mathrm{M}+\mathrm{H}]^{+}\right)$.

IR (ATR): $1724(\mathrm{C}=\mathrm{O}) \mathrm{cm}^{-1} ;{ }^{1} \mathrm{H}-\mathrm{NMR}$ in $\mathrm{CDCl}_{3} \delta$ (ppm): $0.78-0.93$ $\left(6, \mathrm{~m}, \mathrm{C}-\mathrm{CH}_{3}\right), 1.17$ - 1.40 (68, m, C-C $\left.\underline{\mathrm{H}}_{2}-\right), 1.60$ (8, quin, $\left.\mathrm{O}=\mathrm{CCCH}_{2}-, \mathrm{O}-\mathrm{CCH}_{2}-\right), 1.92-2.09\left(8, \mathrm{~m},-\mathrm{CH}=\mathrm{CCH}_{2}-\right), 2.27$ (4, t, $\left.\mathrm{O}=\mathrm{CCH}_{2}-\right), 4.04$ (4, t, O- $\left.\underline{\mathrm{H}}_{2}-\right)$, 5.28 - 5.42 (4, m, - $\left.\overrightarrow{\mathrm{CH}}=\mathrm{C} \underline{\mathrm{H}}-\right)$. MS (ESI): calculated for $\mathrm{C}_{54} \mathrm{H}_{102} \mathrm{O}_{4} 815$, found $m / z 838\left([\mathrm{M}+\mathrm{Na}]^{+}\right)$.

\section{Asymmetric unsaturated diesters:}

$10-3-18$

3-(dec-9-enoyloxy)propyl

IR (ATR): $1738(\mathrm{C}=\mathrm{O}) \mathrm{cm}^{-1} ;{ }^{1} \mathrm{H}-\mathrm{NMR}$ in $\mathrm{CDCl}_{3} \delta$ (ppm): 5.77-5.82 oleate

$\left(1, \mathrm{~m}, \mathrm{CH}_{2}=\mathrm{C} \underline{\mathrm{H}}-\right)$, 5.33-5.36 (2, m, $\left.-\mathrm{CH}=\mathrm{CH}-\right)$, 4.91-5.00 (2, qd, - 
$\left.\mathrm{CH}=\mathrm{C}_{2}\right), 4.13-4.18\left(4, \mathrm{t}, \mathrm{O}-\underline{C}_{2}-\right), 2.27-2.30\left(4, \mathrm{t}, \mathrm{O}=\mathrm{CC} \underline{H}_{2}-\right), 1.98-$ $2.05\left(6 \mathrm{H}, \mathrm{m},-\mathrm{CH}=\mathrm{CC} \underline{\mathrm{H}}_{2}-\right), 1.93-1.97\left(2 \mathrm{H}, \mathrm{m}, \mathrm{O}-\mathrm{CC}_{2}-\right), 1.59-1.63$ (4H, m, O=CCC $\left.\underline{\mathrm{H}}_{2}-\right), 1.26-1.36$ (28, m, C-C $\left.\underline{\mathrm{H}}_{2}-\right), 0.86-0.88$ (3, t, C$\mathrm{CH}_{3}$ ). MS (ESI): calculated for $\mathrm{C}_{31} \mathrm{H}_{56} \mathrm{O}_{4} 493$, found $m / z 511$ ([M + $\left.\mathrm{NH}_{4}\right]^{+}$).

10-4-18 4-(dec-9-enoyloxy)butyl oleate

10-6-18 6-(dec-9-enoyloxy)hexyl oleate

10-9-18 9-(dec-9-enoyloxy)nonyl oleate
IR (ATR): $1735(\mathrm{C}=\mathrm{O}) \mathrm{cm}^{-1} ;{ }^{1} \mathrm{H}-\mathrm{NMR}$ in $\mathrm{CDCl}_{3} \delta$ (ppm): 5.79-5.84 ( $1, \mathrm{~m}, \mathrm{CH}_{2}=\mathrm{C} \underline{\mathrm{H}}-$ ), 5.35-5.37 (2, m, - $\left.\mathrm{CH}=\mathrm{C} \underline{\mathrm{H}}-\right), 4.93-5.02$ (2, qd, $\left.\mathrm{CH}=\mathrm{CH}_{2}\right), 4.09-4.12\left(4, \mathrm{t}, \mathrm{O}-\mathrm{C}_{2}-\right), 2.29-2.32\left(4, \mathrm{t}, \mathrm{O}=\mathrm{CCH}_{2}-\right), 2.00-$ $2.07\left(6 \mathrm{H}, \mathrm{m},-\mathrm{CH}=\mathrm{CCH}_{2}-\right), 1.71-1.74\left(4 \mathrm{H}, \mathrm{m}, \mathrm{O}-\mathrm{CCH}_{2}-\right), 1.60-1.63$ (4H, m, O= $\left.\mathrm{CCCH}_{2}-\right), 1.27-1.42$ (28, m, C- $\left.\mathrm{CH}_{2}-\right), 0.88-0.91$ (3, t, C$\mathrm{CH}_{3}$ ). MS (ESI): calculated for $\mathrm{C}_{32} \mathrm{H}_{58} \mathrm{O}_{4} 507$, found $m / z 525$ ([M + $\left.\mathrm{NH}_{4}\right]^{+}$).

IR (ATR): $1735(\mathrm{C}=\mathrm{O}) \mathrm{cm}^{-1} ;{ }^{1} \mathrm{H}-\mathrm{NMR}$ in $\mathrm{CDCl}_{3} \delta(\mathrm{ppm}):$ 5.79-5.83 (1, m, $\mathrm{CH}_{2}=\mathrm{C}$ $\left.\mathrm{CH}=\mathrm{CH}_{2}\right), 4.05-4.07\left(4, \mathrm{t}, \mathrm{O}-\mathrm{CH}_{2}-\right), 2.27-2.30\left(4, \mathrm{t}, \mathrm{O}=\mathrm{CCH}_{2}-\right), 1.99-$ $2.06\left(6 \mathrm{H}, \mathrm{m},-\mathrm{CH}=\mathrm{CC}_{2_{2}}\right), 1.59-1.65\left(8 \mathrm{H}, \mathrm{m}, \mathrm{O}-\mathrm{CC}_{2_{2}}, \mathrm{O}=\mathrm{CCC} \underline{\mathrm{H}}_{2}-\right.$ ), 1.38-1.40 (4H, m, O-CCC $\left.\underline{H}_{2}-\right), 1.25-1.40\left(28, \mathrm{~m}, \mathrm{C}-\mathrm{C}_{2}-\right), 0.87-$ 0.89 (3, t, C- $\left.\underline{\mathrm{H}}_{3}\right)$. MS (ESI): calculated for $\mathrm{C}_{34} \mathrm{H}_{62} \mathrm{O}_{4} 535$, found $\mathrm{m} / \mathrm{z}$ $553\left(\left[\mathrm{M}+\mathrm{NH}_{4}\right]^{+}\right)$.

IR (ATR): $1735(\mathrm{C}=\mathrm{O}) \mathrm{cm}^{-1} ;{ }^{1} \mathrm{H}-\mathrm{NMR}$ in $\mathrm{CDCl}_{3} \delta(\mathrm{ppm}):$ 5.77-5.82 ( $1, \mathrm{~m}, \mathrm{CH}_{2}=\mathrm{C} \underline{\mathrm{H}}-$ ), 5.32-5.35 (2, m, - $\mathrm{CH}=\mathrm{CH}-$ ) , 4.91-5.00 (2, qd, $\left.\mathrm{CH}=\mathrm{CH}_{2}-\right), 4.03-4.06\left(4, \mathrm{t}, \mathrm{O}-\mathrm{CH}_{2}-\right), 2.26-2.30\left(4, \mathrm{t}, \mathrm{O}=\mathrm{CCH}_{2}-\right)$, 1.98-2.05 (6H, m, $\left.-\mathrm{CH}=\mathrm{CCH}_{2}-\right), 1.58-1.62\left(8 \mathrm{H}, \mathrm{m}, \mathrm{O}-\mathrm{CCH}_{2}-\right.$, $\left.\mathrm{O}=\mathrm{CCCH}_{2}-\right), 1.26-1.38$ (38, m, C- $\left.\mathrm{CH}_{2}-\right), 0.86-0.89$ (3, t, $\left.\mathrm{C}-\mathrm{CH}_{3}\right) . \mathrm{MS}$ (ES): calculated for $\mathrm{C}_{37} \mathrm{H}_{68} \mathrm{O}_{4} 577$, found $m / z 578\left([\mathrm{M}+\mathrm{H}]^{+}\right)$.

\begin{tabular}{|c|c|c|}
\hline $18-3-22$ & $\begin{array}{l}\text { 3-(oleoyloxy)propyl }(Z)- \\
\text { docos-13-enoate }\end{array}$ & $\begin{array}{l}\mathrm{IR}(\mathrm{ATR}): 1738(\mathrm{C}=\mathrm{O}) \mathrm{cm}^{-1} ;{ }^{1} \mathrm{H}-\mathrm{NMR} \text { in } \mathrm{CDCl}_{3} \delta(\mathrm{ppm}): 0.87-0.89 \\
\left(6, \mathrm{~m}, \mathrm{C}-\mathrm{CH}_{3}\right), 1.26-1.30\left(48, \mathrm{~m}, \mathrm{C}-\mathrm{C}_{\mathrm{H}_{2}}-\right), 1.60-1.63(4, \mathrm{~m}, \\
\left.\mathrm{O}=\mathrm{CCC} \underline{H}_{2}-\right), 1.94-1.97\left(2, \mathrm{~m}, \mathrm{O}-\mathrm{CC}_{\mathrm{H}_{2}}-\right), 1.99-2.03(8, \mathrm{~m},- \\
\left.\mathrm{CH}=\mathrm{CC} \underline{\mathrm{H}}_{2}-\right), 2.28-2.31\left(4, \mathrm{t}, \mathrm{O}=\mathrm{CC} \underline{\mathrm{H}}_{2}-\right), 4.14-4.16\left(4, \mathrm{~m}, \mathrm{O}-\mathrm{CH}_{2}-\right), \\
5.31-5.38(4, \mathrm{~m},-\mathrm{CH}=\mathrm{CH}-) .\end{array}$ \\
\hline $18-4-22$ & $\begin{array}{l}\text { 4-(oleoyloxy)butyl }(Z) \text { - } \\
\text { docos-13-enoate }\end{array}$ & 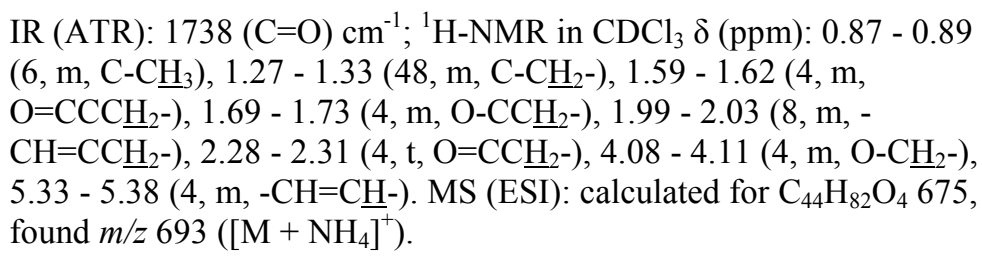 \\
\hline $18-6-22$ & $\begin{array}{l}\text { 6-(oleoyloxy)hexyl (Z)- } \\
\text { docos-13-enoate }\end{array}$ & 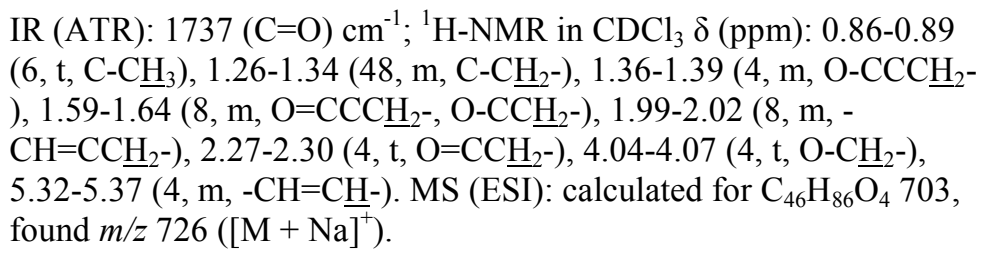 \\
\hline $18-9-22$ & $\begin{array}{l}\text { 9-(oleoyloxy)nonyl (Z)- } \\
\text { docos-13-enoate }\end{array}$ & 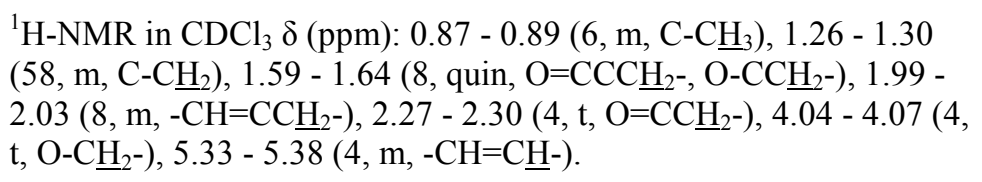 \\
\hline $18-10-22$ & $\begin{array}{l}\text { 10-(oleoyloxy)decyl }(Z)- \\
\text { docos-13-enoate }\end{array}$ & $\begin{array}{l}\text { IR (ATR): } 1728(\mathrm{C}=\mathrm{O}) \mathrm{cm}^{-1} ;{ }^{1} \mathrm{H}-\mathrm{NMR} \text { in } \mathrm{CDCl}_{3} \delta(\mathrm{ppm}): 0.86-0.89 \\
\left(6, \mathrm{~m}, \mathrm{C}-\mathrm{CH}_{3}\right), 1.26-1.29\left(60, \mathrm{~m}, \mathrm{C}-\mathrm{C}_{2}{ }^{-}\right), 1.58-1.62(8, \mathrm{~m},\end{array}$ \\
\hline
\end{tabular}


$\left.\mathrm{O}=\mathrm{CCC}_{\mathrm{H}_{2}}-, \mathrm{O}-\mathrm{CC}_{\mathrm{H}_{2}}-\right), 1.99-2.02\left(8, \mathrm{~m}, \mathrm{C}=\mathrm{CC}_{\mathrm{H}_{2}}-\right), 2.26-2.29$ (4, t, $\left.\mathrm{O}=\mathrm{CC} \underline{H}_{2}-\right), 4.03-4.06\left(4, \mathrm{t}, \mathrm{O}-\mathrm{C}_{2}{ }^{-}\right), 5.32-5.37(4, \mathrm{~m},-\mathrm{CH}=\mathrm{C} \underline{H}-) . \mathrm{MS}$ (ESI): calculated for $\mathrm{C}_{50} \mathrm{H}_{94} \mathrm{O}_{4} 759$, found $m / z 782\left([\mathrm{M}+\mathrm{Na}]^{+}\right)$.

\section{Asymmetric saturated-unsaturated \\ diesters:}

16-3-18 3-(palmitoyloxy)propyl oleate

16-4-18 4-(palmitoyloxy)butyl oleate

$16-6-18$

6-(palmitoyloxy)hexyl oleate

16-10-18 10-(palmitoyloxy)decyl oleate
IR (ATR): $1717(\mathrm{C}=\mathrm{O}) \mathrm{cm}^{-1} ;{ }^{1} \mathrm{H}-\mathrm{NMR}$ in $\mathrm{CDCl}_{3} \delta(\mathrm{ppm}): 0.86-0.89$ (6, m, C-C $\left.\underline{H}_{3}\right), 1.25-1.29$ (44, m, C-C $\left.\underline{H}_{2}-\right), 1.59-1.62(4, \mathrm{~m}$, $\left.\mathrm{O}=\mathrm{CCC}_{2_{2}}-\right), 1.93-1.97\left(2, \mathrm{~m}, \mathrm{O}-\mathrm{CC}_{2_{2}}\right), 1.98-2.02\left(4, \mathrm{~m}, \mathrm{C}=\mathrm{CC}_{\mathrm{H}_{2}}{ }^{-}\right.$ ), 2.27-2.30 (4, t, $\left.\mathrm{O}=\mathrm{CCH}_{2}-\right), 4.13-4.15$ (4, t, O- $\left.\mathrm{C}_{2}-\right), 5.32-5.35$ (2, $\mathrm{m},-\mathrm{CH}=\mathrm{CH}-$-). $\mathrm{MS}$ (ESI): calculated for $\mathrm{C}_{37} \mathrm{H}_{70} \mathrm{O}_{4} 579$, found $\mathrm{m} / z 602$ $\left([\mathrm{M}+\mathrm{Na}]^{+}\right)$.

IR (ATR): $1725(\mathrm{C}=\mathrm{O}) \mathrm{cm}^{-1} ;{ }^{1} \mathrm{H}-\mathrm{NMR}$ in $\mathrm{CDCl}_{3} \delta$ (ppm): $0.87-0.89$ (6, m, C-C $\left.\underline{H}_{3}\right), 1.25-1.30$ (44, m, C-C $\left.\underline{H}_{2}-\right), 1.60-1.63$ (4, m,

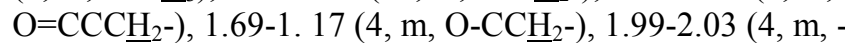
$\left.\mathrm{CH}=\mathrm{CC}_{2_{2}}\right), 2.28-2.31\left(4, \mathrm{t}, \mathrm{O}=\mathrm{CC}_{\mathrm{H}_{2}}-\right), 4.08-4.11\left(4, \mathrm{t}, \mathrm{O}-\mathrm{C}_{2}-\right)$, 5.33-5.36 (2, m, $-\mathrm{CH}=\mathrm{C} \underline{\mathrm{H}}-)$. MS (ESI): calculated for $\mathrm{C}_{38} \mathrm{H}_{72} \mathrm{O}_{4} 593$, found $\mathrm{m} / z 616\left([\mathrm{M}+\mathrm{Na}]^{+}\right)$.

IR (ATR): $1727(\mathrm{C}=\mathrm{O}) \mathrm{cm}^{-1} ;{ }^{1} \mathrm{H}-\mathrm{NMR}$ in $\mathrm{CDCl}_{3} \delta$ (ppm): 0.86-0.88 (6, m, C-C $\left.\underline{\mathrm{H}}_{3}\right), 1.24-1.29$ (44, m, C-C $\left.\underline{\mathrm{H}}_{2}-\right), 1.36-1.39$ (4, m, O-

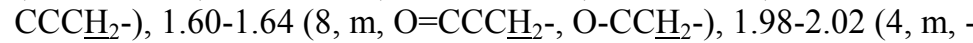
$\left.\mathrm{CH}=\mathrm{CC}_{2}-\right), 2.26-2.29\left(4, \mathrm{t}, \mathrm{O}=\mathrm{CC}_{2}-\right), 4.04-4.06\left(4, \mathrm{t}, \mathrm{O}-\mathrm{C}_{2}-\right)$, 5.32-5.34 (2, m, - $\mathrm{CH}=\mathrm{CH}-$ ). MS (ESI): calculated for $\mathrm{C}_{40} \mathrm{H}_{76} \mathrm{O}_{4} 621$, found $\mathrm{m} / z 644\left([\mathrm{M}+\mathrm{Na}]^{+}\right)$.

IR (ATR): $1729(\mathrm{C}=\mathrm{O}) \mathrm{cm}^{-1} ;{ }^{1} \mathrm{H}-\mathrm{NMR}$ in $\mathrm{CDCl}_{3} \delta$ (ppm): 0.87-0.89 (6, m, C-C $\left.\underline{H}_{3}\right), 1.25-1.30$ (56, m, C-C $\left.\underline{\mathrm{H}}_{2}-\right), 1.57-1.64$ (8, m, $\left.\mathrm{O}=\mathrm{CCCH}_{2}-, \mathrm{O}-\mathrm{CCH}_{2}-\right), 1.99-2.03\left(4, \mathrm{~m},-\mathrm{CH}=\mathrm{CCH}_{2}-\right), 2.27-2.30$ (4, $\left.\mathrm{t}, \mathrm{O}=\mathrm{CCH}_{2}-\right), 4.04-4.07$ (4, t, O- $\left.\mathrm{CH}_{2}-\right), 5.33-5.36(2, \mathrm{~m},-\mathrm{CH}=\mathrm{CH}-)$. MS (ESI): calculated for $\mathrm{C}_{44} \mathrm{H}_{84} \mathrm{O}_{4} 677$, found $\mathrm{m} / z 700\left([\mathrm{M}+\mathrm{Na}]^{+}\right)$.

$\begin{array}{ll}\text { 16-3-22 } & \text { (palmitoyloxy)propyl } \\ & \text { (Z)-docos-13-enoate }\end{array}$

16-4-22 4-(palmitoyloxy)butyl (Z)-docos-13-enoate

$16-6-22$

6-(palmitoyloxy)hexyl (Z)-docos-13-enoate

$16-9-22$
IR (ATR): $1725(\mathrm{C}=\mathrm{O}) \mathrm{cm}^{-1} ;{ }^{1} \mathrm{H}-\mathrm{NMR}$ in $\mathrm{CDCl}_{3} \delta(\mathrm{ppm}): 0.86-0.89$ (6, t, C-C $\left.\underline{H}_{3}\right), 1.25-1.28$ (52, m, C-C $\left.\underline{H}_{2}-\right), 1.58-1.63$ (4, m, $\left.\mathrm{O}=\mathrm{CCC}_{2}-\right), 1.93-1.97\left(2, \mathrm{~m}, \mathrm{O}-\mathrm{CC} \underline{H}_{2}-\right), 1.99-2.02(4, \mathrm{~m},-$ $\left.\mathrm{CH}=\mathrm{CC}_{2}{ }_{2}^{-}\right), 2.27-2.30$ (4, t, $\left.\mathrm{O}=\mathrm{CC}_{\mathrm{H}_{2}}-\right), 4.13-4.15\left(4, \mathrm{t}, \mathrm{O}-\mathrm{C}_{2}-\right)$, 5.33-5.35 (2, m, - $\mathrm{CH}=\mathrm{CH}-$-). MS (ESI): calculated for $\mathrm{C}_{41} \mathrm{H}_{78} \mathrm{O}_{4} 635$, found $m / z 658\left([\mathrm{M}+\mathrm{Na}]^{+}\right)$.

IR (ATR): $1726(\mathrm{C}=\mathrm{O}) \mathrm{cm}^{-1} ;{ }^{1} \mathrm{H}-\mathrm{NMR}$ in $\mathrm{CDCl}_{3} \delta(\mathrm{ppm}): 0.86-0.89$ (6, t, C-C $\left.\underline{H}_{3}\right), 1.25-1.28\left(52, \mathrm{~m}, \mathrm{C}-\mathrm{C}_{2}-\right), 1.58-1.64(4, \mathrm{~m}$, $\left.\mathrm{O}=\mathrm{CCC} \underline{\mathrm{H}}_{2}-\right), 1.68-1.71\left(4, \mathrm{~m}, \mathrm{O}-\mathrm{CC} \underline{\mathrm{H}}_{2}-\right), 1.99-2.03(4, \mathrm{~m},-$ $\mathrm{CH}=\mathrm{CC}_{2_{2}}$ ), 2.27-2.30 (4, t, $\left.\mathrm{O}=\mathrm{CC}_{\mathrm{H}_{2}}\right)$ ), 4.09 (4, m, O-C $\left.\underline{\mathrm{H}}_{2}-\right)$, 5.33$5.35(2, \mathrm{~m},-\mathrm{CH}=\mathrm{CH}-)$. MS (ESI): calculated for $\mathrm{C}_{42} \mathrm{H}_{80} \mathrm{O}_{4} 649$, found $m / z 672\left([\mathrm{M}+\mathrm{Na}]^{+}\right)$.

IR (ATR): $1725(\mathrm{C}=\mathrm{O}) \mathrm{cm}^{-1} ;{ }^{1} \mathrm{H}-\mathrm{NMR}$ in $\mathrm{CDCl}_{3} \delta(\mathrm{ppm}): 0.87-0.89$ (6, t, C-C $\left.\underline{H}_{3}\right), 1.25-1.29$ (52, m, C-C $\left.\underline{H}_{2}-\right), 1.37-1.40$ (4, m, O-CCC $\underline{H}_{2-}$

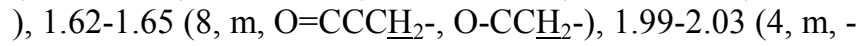
$\left.\mathrm{CH}=\mathrm{CC}_{\mathrm{H}_{2}}-\right), 2.27-2.30$ (4, t, $\left.\mathrm{O}=\mathrm{CC}_{\mathrm{H}_{2}}-\right), 4.05$ - 4.07 (4, m, O-C $\underline{\mathrm{H}}_{2}-$ ), 5.34-5.36 (2, m, - $\mathrm{CH}=\mathrm{CH}-$ ). MS (ESI): calculated for $\mathrm{C}_{44} \mathrm{H}_{84} \mathrm{O}_{4} 677$, found $m / z 700\left([\mathrm{M}+\mathrm{Na}]^{+}\right)$.

IR (ATR): $1735(\mathrm{C}=\mathrm{O}) \mathrm{cm}^{-1} ;{ }^{1} \mathrm{H}-\mathrm{NMR}$ in $\mathrm{CDCl}_{3} \delta$ (ppm): 0.87-0.89 (6, t, C-C $\left.\underline{H}_{3}\right), 1.25-1.30$ (62, m, C-C $\left.\underline{\mathrm{H}}_{2}-\right), 1.60-1.64$ (8, m, O-CC $\underline{H}_{2}$, 
$\left.\mathrm{O}=\mathrm{CCC}_{2}{ }_{2}-\right), 1.99-2.03\left(4, \mathrm{~m},-\mathrm{CH}=\mathrm{CCH}_{2}-\right), 2.27-2.30(4, \mathrm{t}$, $\left.\mathrm{O}=\mathrm{CC}_{2_{2}}\right)$, $4.04-4.07$ (4, m, O-C $\left.\underline{\mathrm{H}}_{2}-\right), 5.34-5.36(2, \mathrm{~m},-\mathrm{CH}=\mathrm{C} \underline{\mathrm{H}}-)$. MS (ESI): calculated for $\mathrm{C}_{47} \mathrm{H}_{90} \mathrm{O}_{4} 719$, found $m / z 720\left([\mathrm{M}+\mathrm{H}]^{+}\right)$.

16-10-22 10-(palmitoyloxy)decyl (Z)-docos-13-enoate
IR (ATR): $1728(\mathrm{C}=\mathrm{O}) \mathrm{cm}^{-1} ;{ }^{1} \mathrm{H}-\mathrm{NMR}$ in $\mathrm{CDCl}_{3} \delta(\mathrm{ppm}): 0.87-0.89$

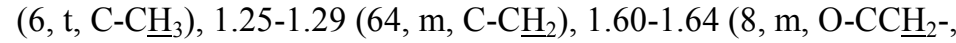
$\left.\mathrm{O}=\mathrm{CCC} \underline{H}_{2}-\right), 1.99-2.03$ (4, m, $\left.-\mathrm{CH}=\mathrm{CCH}_{2}-\right), 2.27-2.30$ (4, t, $\left.\mathrm{O}=\mathrm{CC}_{2}-\right), 4.04-4.07$ (4, m, O-C $\left.\underline{\mathrm{H}}_{2}-\right), 5.34-5.36$ (2, m, $\left.-\mathrm{CH}=\mathrm{CH}-\right)$. MS (ESI): calculated for $\mathrm{C}_{48} \mathrm{H}_{92} \mathrm{O}_{4} 733$, found $m / z 734\left([\mathrm{M}+\mathrm{H}]^{+}\right)$. 
<smiles>[R]C(=O)OC1(C)CC1(OC([R])=O)OC([R])=O</smiles>

Scheme S.1: Synthesis of symmetric diesters from fatty acids and diols.

Stage 1:

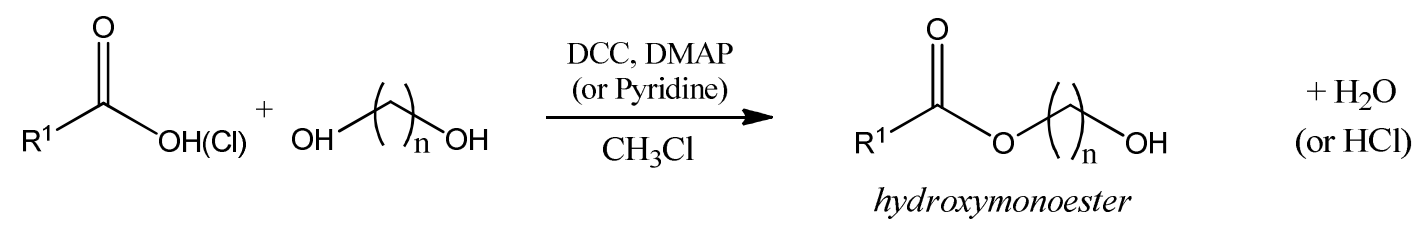

Stage 2:

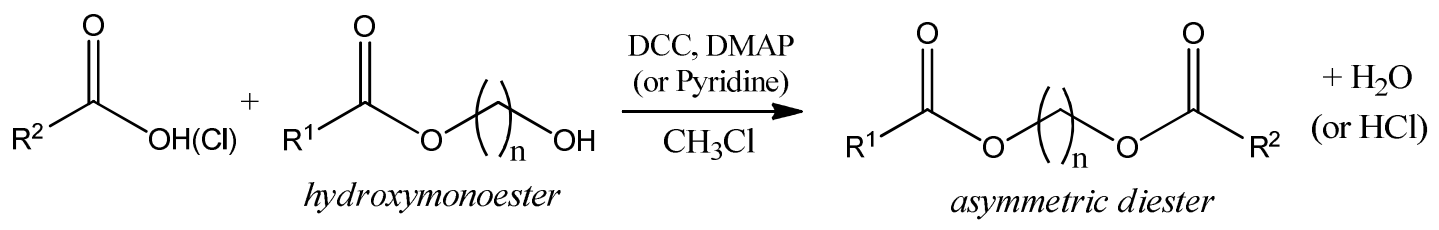

Scheme S.2: Showing the two-stage syntheses of asymmetric diesters. Given in brackets are the reaction conditions and products when fatty acid chlorides are used instead of fatty acids. 
(a) $10-n-10$

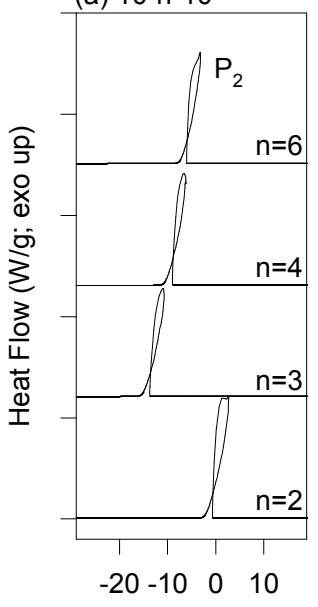

(b) $10-n-18$

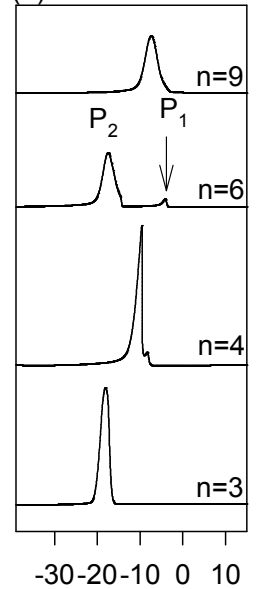

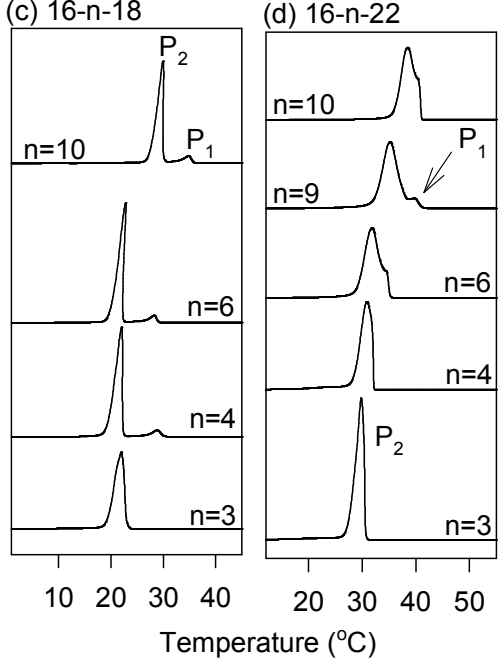

(e) $18-n-22$

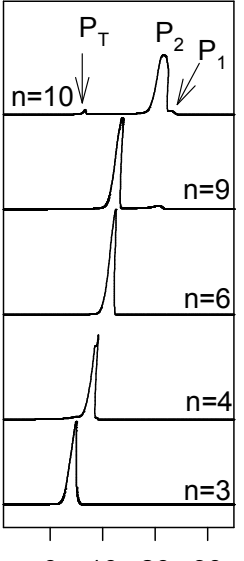

$\begin{array}{llll}0 & 10 & 20 & 30\end{array}$ (f) $22-n-22$

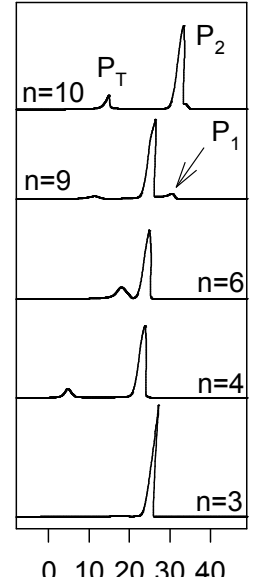

Figure S.1: DSC cooling $\left(3{ }^{\circ} \mathrm{C} / \mathrm{min}\right)$ thermograms obtained for the linear aliphatic diesters. $\mathrm{P}_{1}, \mathrm{P}_{2}$ and $\mathrm{P}_{\mathrm{T}}$ are the lead, main, and tail peak events, respectively.

(a) $10-n-10$

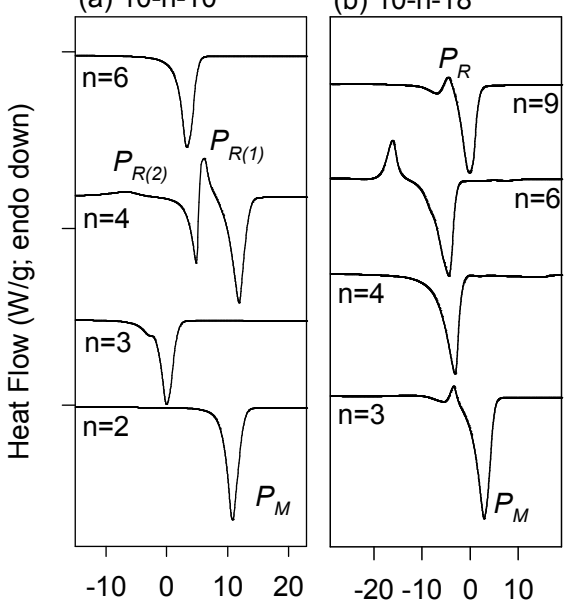

(c) $16-n-18$

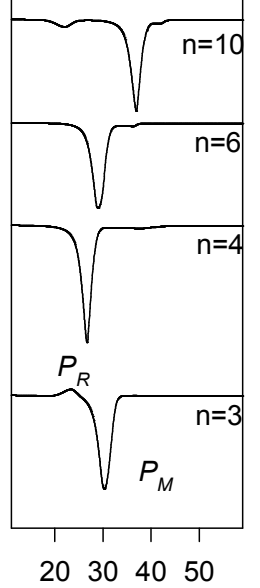

(d) 16-n-22

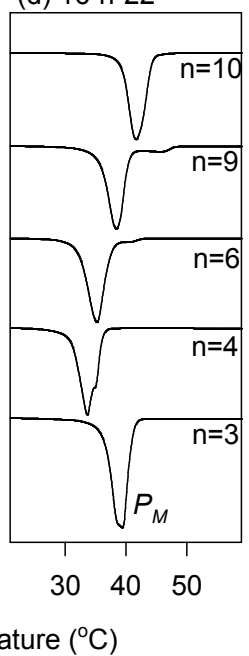

(e) $18-n-22$

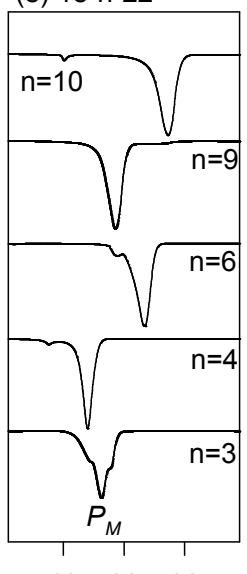

$10 \quad 20 \quad 30$

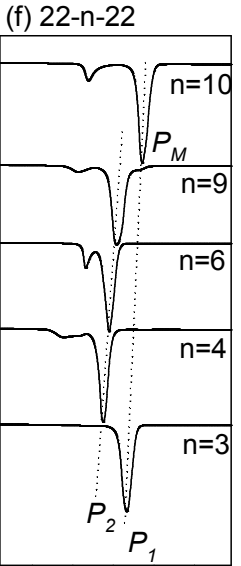

1020304050

Temperature $\left({ }^{\circ} \mathrm{C}\right)$

Figure S.2: DSC heating $\left(3{ }^{\circ} \mathrm{C} / \mathrm{min}\right)$ thermograms of linear aliphatic diesters. $\mathrm{P}_{\mathrm{M}}$ shows the main endotherm, and $\mathrm{P}_{\mathrm{R}}$ shows the exotherm from the crystallization mediated by melt. $\mathrm{P}_{1}$ and $\mathrm{P}_{2}$ show the melting of the two most stable phases in order of decreasing stability. 

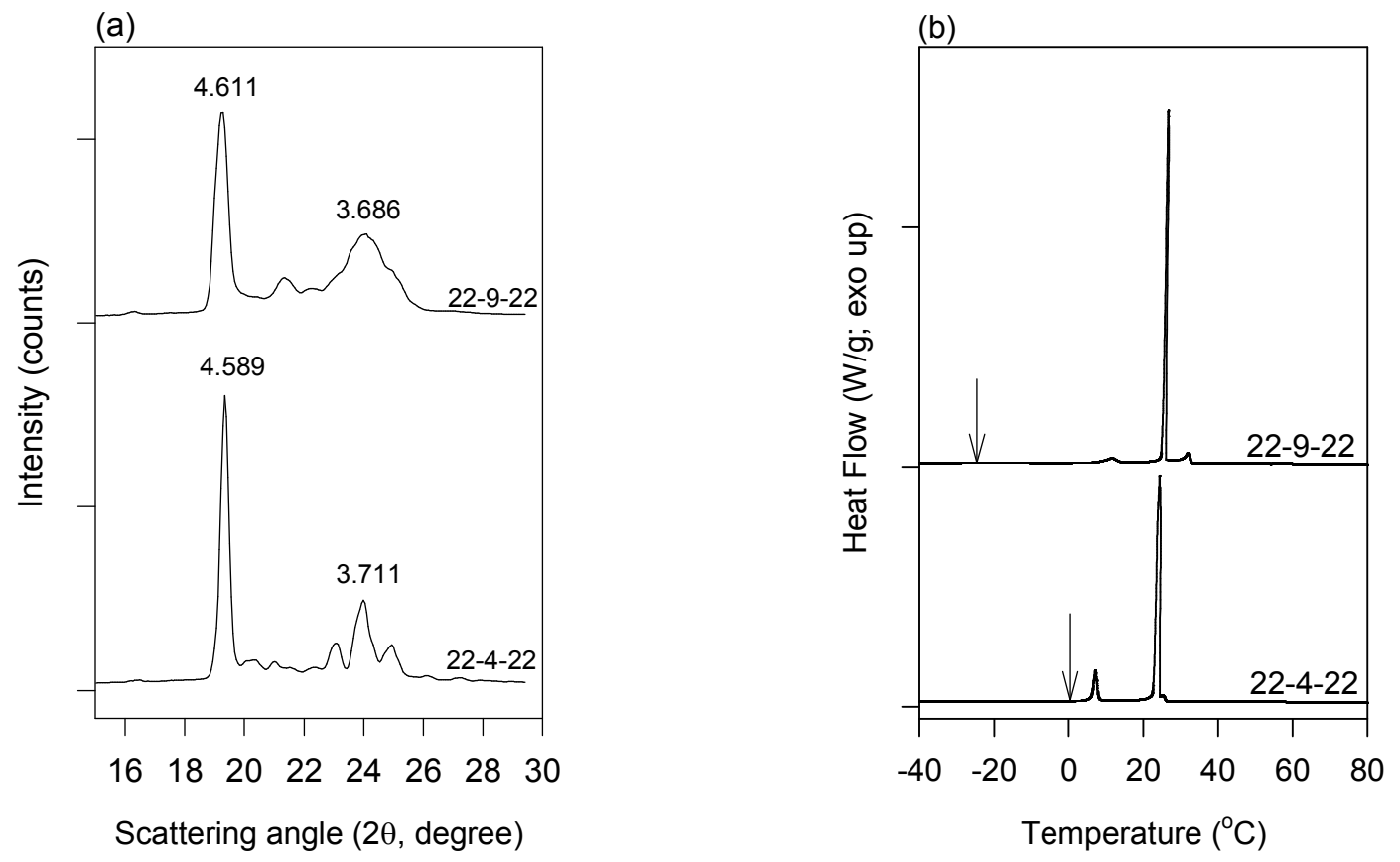

Figure S.3: (a) WAXD plots for $22-4-22$ and $22-9-22$ diesters obtained at $0{ }^{\circ} \mathrm{C}$ and $-25{ }^{\circ} \mathrm{C}$, respectively using a cooling rate of $1{ }^{\circ} \mathrm{C} / \mathrm{min}$; d-spacing values, in Angstroms, are given at the top of the characteristic peaks. (b) DSC cooling profiles of 22-4-22 and 22-9-22 obtained using the same thermal protocol as was used for determination of the WAXD profiles; arrows show the temperature at which the WAXD was taken. 


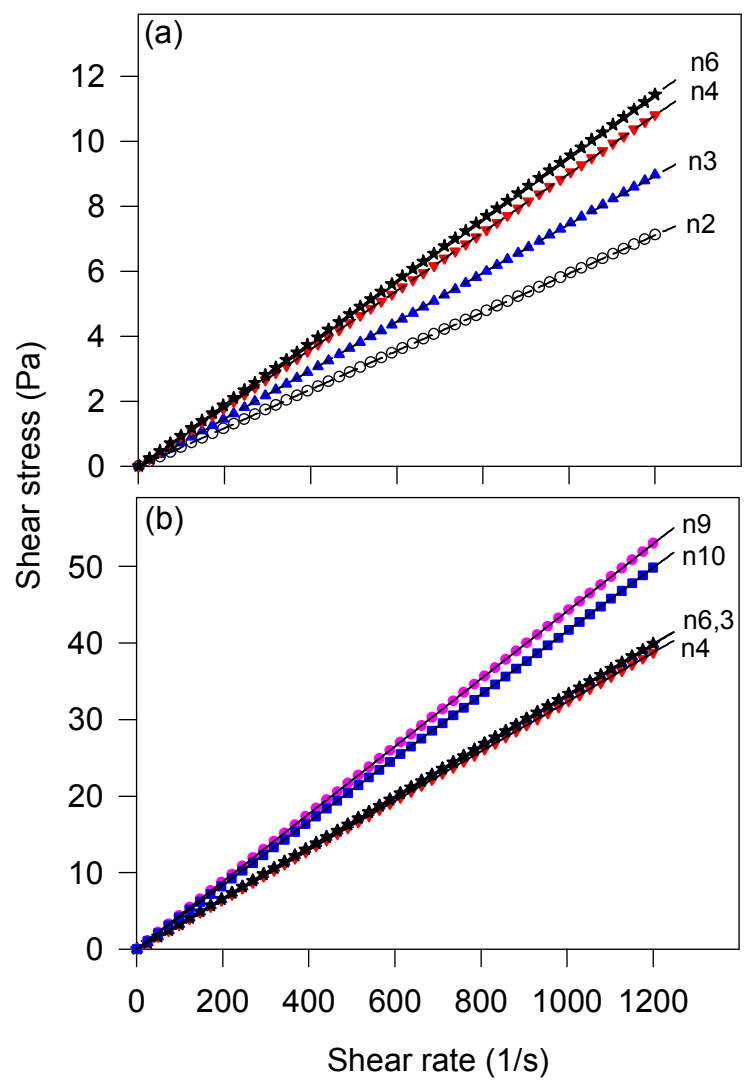

Figure S.4: Shear rate versus shear stress plots of representative diester series measured at $40{ }^{\circ} \mathrm{C}$ (a) 10-n-10 and (b) 22-n-22 diesters. Dashed lines are linear fits $\left(r^{2}>0.9975\right)$ to the HerschelBulkley model. 
(a)

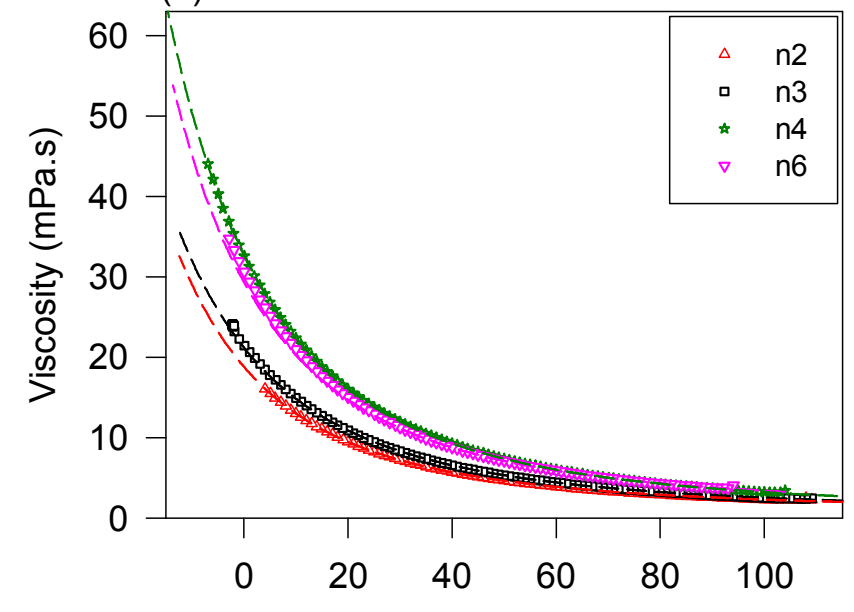

(b)

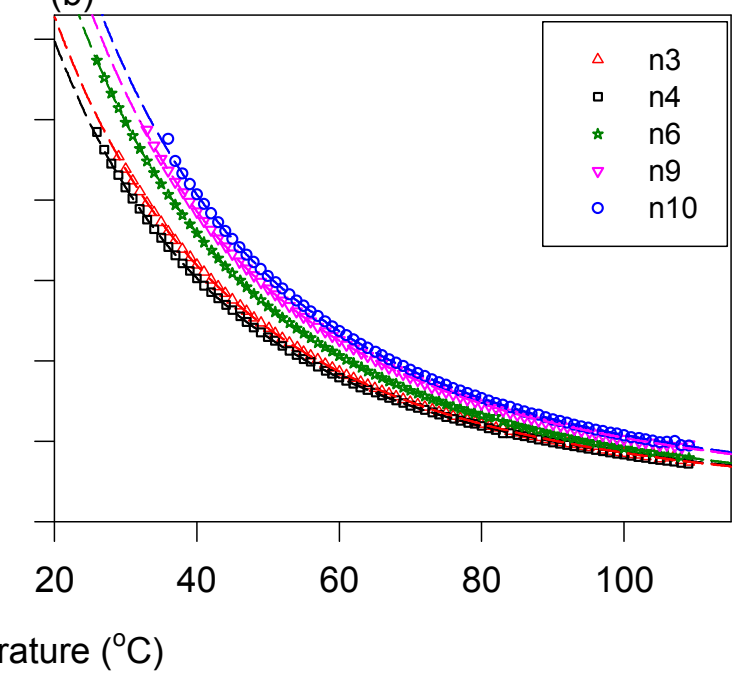

Figure S.5: Temperature-Viscosity profiles of representative diester series (a) 10-n-10; and (b) 22-n-22. Cooling rate $3{ }^{\circ} \mathrm{C} / \mathrm{min}$. Dashed lines are guides for the eye. 